OPEN ACCESS

Edited by:

Christian Gonzalez-Billault,

University of Chile, Chile

Reviewed by:

Alvaro Glavic,

Center for Genome Regulation

(CGR), Chile

Haijun TU,

Hunan University, China

*Correspondence:

Allison Birnbaum

abirnba1@uab.edu

Hua Bai

hbai@iastate.edu

Received: 30 May 2020 Accepted: 04 December 2020 Published: 27 January 2021

Citation:

Birnbaum A, Sodders M, Bouska M Chang K, Kang P, MCNeill E and Bai H (2021) FOXO Regulates Neuromuscular Junction Homeostasis During Drosophila Aging. Front. Aging Neurosci. 12:567861. doi: 10.3389/fnagi.2020.567861

\section{FOXO Regulates Neuromuscular Junction Homeostasis During Drosophila Aging}

\author{
Allison Birnbaum ${ }^{1,2 *}$, Maggie Sodders ${ }^{1}$, Mark Bouska ${ }^{1}$, Kai Chang ${ }^{1}$, Ping Kang ${ }^{1}$, \\ Elizabeth $\mathrm{McNeill}^{3}$ and Hua Bai ${ }^{1 *}$ \\ ${ }^{1}$ Department of Genetics, Development, and Cell Biology, lowa State University, Ames, IA, United States, ${ }^{2}$ Department of \\ Cell, Developmental and Integrative Biology, University of Alabama Birmingham, Birmingham, AL, United States, \\ ${ }^{3}$ Department of Food Science and Human Nutrition, lowa State University, Ames, IA, United States
}

The transcription factor foxo is a known regulator of lifespan extension and tissue homeostasis. It has been linked to the maintenance of neuronal processes across many species and has been shown to promote youthful characteristics by regulating cytoskeletal flexibility and synaptic plasticity at the neuromuscular junction (NMJ). However, the role of foxo in aging neuromuscular junction function has yet to be determined. We profiled adult Drosophila foxo- null mutant abdominal ventral longitudinal muscles and found that young mutants exhibited morphological profiles similar to those of aged wild-type flies, such as larger bouton areas and shorter terminal branches. We also observed changes to the axonal cytoskeleton and an accumulation of late endosomes in foxo null mutants and motor neuron-specific foxo knockdown flies, similar to those of aged wild-types. Motor neuron-specific overexpression of foxo can delay age-dependent changes to NMJ morphology, suggesting foxo is responsible for maintaining NMJ integrity during aging. Through genetic screening, we identify several downstream factors mediated through foxo-regulated NMJ homeostasis, including genes involved in the MAPK pathway. Interestingly, the phosphorylation of p38 was increased in the motor neuron-specific foxo knockdown flies, suggesting foxo acts as a suppressor of p38/MAPK activation. Our work reveals that foxo is a key regulator for NMJ homeostasis, and it may maintain NMJ integrity by repressing MAPK signaling.

Keywords: FOXO, NMJ, MAPK, p38, Rab7, late endosome, aging

\section{INTRODUCTION}

Aging involves the progressive functional decline of cellular mechanisms and tissue integrity (Rose, 1994; Partridge and Barton, 1996; Lopez-Otin et al., 2013). In the adult, this results in a gradual decline of synaptic contacts to skeletal muscle tissue, resulting in a loss of strength and muscle mass (Hall and Sanes, 1993). The neuromuscular junction (NMJ) serves as the synaptic interface between the branched terminals of motor neurons and the skeletal muscle fibers (Punga and Ruegg, 2012). The NMJ is highly dynamic in response to cellular signals and stressors, and a dysregulation of molecular processes in both the pre- and post-synaptic regions can lead to the onset of neurodegenerative diseases (Gonzalez-Freire et al., 2014; Monani and De Vivo, 2014). With aging there is impairment of regulatory systems such as autophagy and redox homeostasis 
at the NMJ, which can lead to reactive oxygen species (ROS) accumulation followed by organelle damage and cell death (Li et al., 2018; Stefanatos and Sanz, 2018). Synaptic plasticity, which allows for the maintenance of functional activity to protect against degeneration, can decline during aging, resulting in decreased neuronal responsiveness and synaptic deterioration (Bergado and Almaguer, 2002; Kempsell and Fieber, 2015; Wagner et al., 2015).

The Drosophila NMJ synapse utilizes glutamate as the primary neurotransmitter and has postsynaptic densities that behave similarly to the AMPA-type receptors in the vertebrate central nervous system (Menon et al., 2013). The Drosophila neuromuscular junction system has been well-stereotyped in larvae, but has only more recently begun to be characterized in the adult fly (Rivlin et al., 2004; Hebbar et al., 2006; Beramendi et al., 2007; Wagner et al., 2015; Lopez-Arias et al., 2017). These studies have also discovered a number of morphological changes that occur at the NMJ under normal aging conditions and can aid in understanding the mechanisms involved in age-dependent neuronal functional decline.

The foxo transcription factor family plays an important role in metabolism and development as well as stress resistance and lifespan (Accili and Arden, 2004; Greer and Brunet, 2005). foxo has long been characterized as a "longevity gene" and as a modulator of protein homeostasis (Kenyon et al., 1993; Martins et al., 2016). FOXO proteins can also function as regulators of neuronal homeostasis (Paik et al., 2009; Kim and Webb, 2017; Schaffner et al., 2018). FOXO-mediated pathways permit neuronal plasticity, which have downstream implications on cellular behavior and neuronal morphology (McLaughlin and Broihier, 2018). Drosophila FOXO (dFOXO, hereafter foxo) promotes cytoskeletal dynamics at the neuromuscular junction during larval development, where it responds to stimulation to allow rapid structural reorganization. In the Drosophila larvae NMJ, loss of foxo results in increased stability of microtubules through the upregulation of kinesin motor protein regulator Paverotti that also controls axon growth (Nechipurenko and Broihier, 2012; McLaughlin et al., 2016). In mammals, certain foxo isoforms show transcriptional regulation of cytoskeletal genes during development (de la Torre-Ubieta et al., 2010). FOXOs are also implicated in regulating autophagy in neurons under Parkinson's disease models (Xu et al., 2011; Pino et al., 2014; Schaffner et al., 2018). It has also been shown that aging results in the activation of pro-inflammatory signals and proteotoxic stress in mice brains, and the depletion of neuronal FOXOs causes premature occurrences of these events and induces motor function impairment (Hwang et al., 2018). Additionally, loss of foxo impacts synaptic function by reducing neurotransmitter release in both larval and adult drosophila neuromuscular junctions (Howlett et al., 2008; Mahoney et al., 2016). Aged synapses also show a reduction in evoked response and neurotransmission (Segal, 1982; Porras and Mora, 1995; Liu et al., 2013). These studied indicate that foxo is an important regulator of motor neuron function, and that foxo impacts the functional and structural integrity of the neuromuscular junction.
Our current knowledge of foxo supports the hypothesis that foxo activity promotes youthful NMJ morphology and provides necessary regulation of pathways involved in maintaining synaptic integrity and plasticity. However, how foxo impacts functional regulation of the NMJ during aging remains to be elucidated. Here, we show that loss of foxo causes morphological alterations to the NMJ in young adult flies and shares phenotypic characteristics with those of aging control organisms. We find that knocking down foxo specifically in the motor neuron results in changes to microtubule morphology and an upregulation of late endocytic vesicles in the nervous system. These events are also observed in middle-aged flies, indicating loss of foxo may lead to a disruption of motor neuron homeostasis. Overexpression of foxo in the motor neuron delays these agerelated changes to NMJ morphology. Additionally, we identified potential pathways downstream of foxo that may promote aging and decline in synaptic function in the adult motor neurons. These findings provide insight into the role foxo plays in maintaining morphological and synaptic plasticity at the aging neuromuscular junction.

\section{RESULTS}

\section{Loss of Foxo Causes Morphological Changes to Bouton Size and Branch Length That Correspond to Aging}

Normal aging causes alterations to the morphology of boutons and branches at the adult Drosophila neuromuscular junction (Beramendi et al., 2007; Wagner et al., 2015; Lopez-Arias et al., 2017). Based on previous literature, we selected three time points post eclosion, 6, 25, and 40 days to represent young, middle aged, and old adult flies. We visualized the A3 abdominal ventral longitudinal muscle (VLM) segment using immunofluorescence staining in female flies. We stained flayed adult abdominal pelts with a monoclonal antibody against bruchpilot (BRP) to mark active zones (AZs) and an antibody against horseradish peroxidase (HRP) to visualize neuronal tissue (Figure 1A). To obtain overall bouton number, we used an antibody against Disclarge (Dlg) to mark post-synaptic regions. Using the two NMJ's present at each A3, we quantified the six metrics characterized by Wagner et al. (2015) and found a significant difference between ages for several metrics, as expected. A significant decrease in branch length was observed with aging, along with a decrease in bouton number (Figure 1B, Table 1). We also found an expansion of bouton area with age, and this corresponded to an increase in number of AZs per bouton (Figures 1C,D, Table 1). However, the number of AZs observed in a $100 \mathrm{um}^{2}$ region did not change between ages, which has been shown to remain constant during aging (Table 1, Wagner et al., 2015).

We next examined how the loss of foxo effects NMJ morphology in the adult. We used the hypomorphic allele foxo ${ }^{21}$ and dissected the VLM of 1-week post eclosion animals and characterized the A3 muscle as described previously (Figure 1A). We found that foxo mutants had significantly larger bouton areas and shorter branches than their 1-week 
A
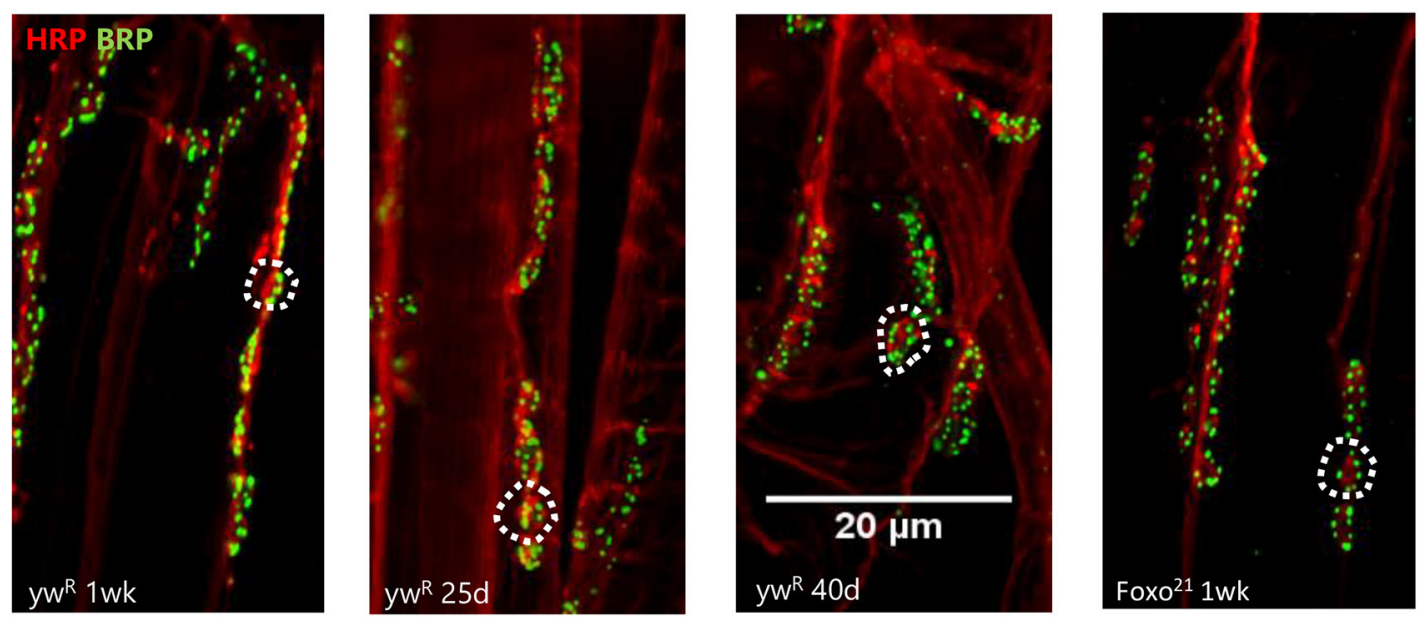

B

Average Branch Length

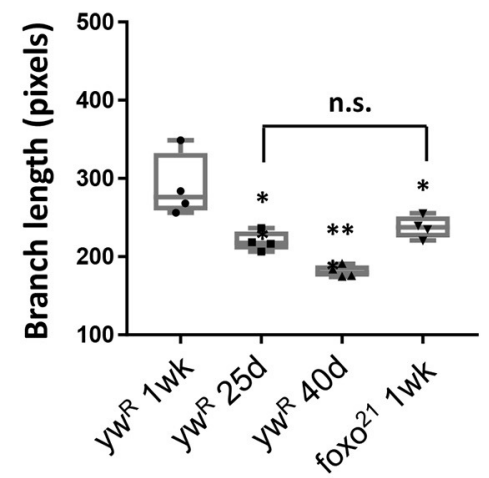

C Average Bouton Area

D
Active zone/bouton
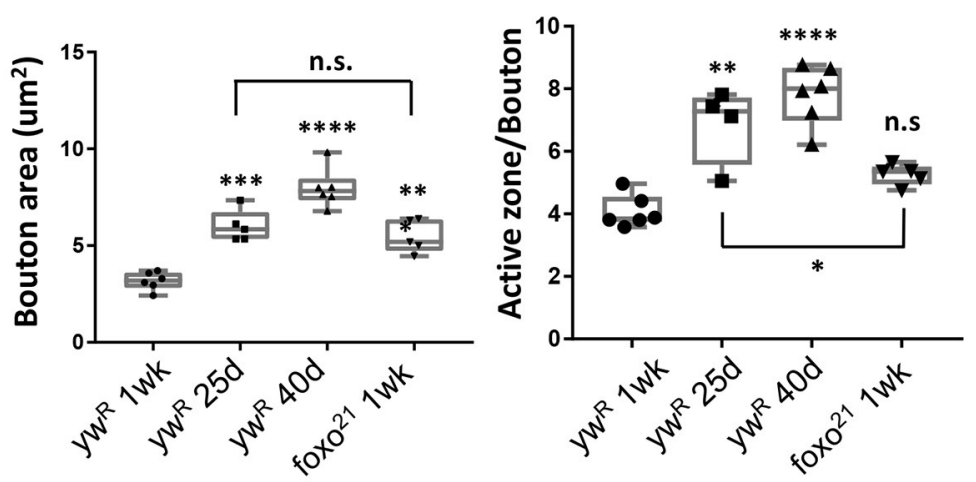

FIGURE 1 | Loss of FOXO results in middle-aged NMJ morphology in the abdominal ventral muscle. (A) Representative images of the Abdominal VLM A3 segment for axon terminals with indicated genotypes and ages colabeled with Anti-BRP and anti-HRP. Bar $20 \mu$ M. (B) Average branch length. ${ }^{\star} P<0.05,{ }^{\star \star} P<0.01$. Bouton area for 1 -week foxo flies is significantly larger than 1 -week controls, but is comparable to 25 -day control flies. ${ }^{* \star} P<0.01$. (C) Average bouton area ${ }^{\star \star} P<0.01$, ${ }^{\star \star *} P>$ $0.001,{ }^{* \star \star \star} P<0.0001$. (D) Quantification of changes in active zone number per bouton. ${ }^{\star} P<0.05,{ }^{\star \star} P<0.01,{ }^{\star \star \star} P<0.001 . y w R 1 \mathrm{wk} n=6 ; 25 \mathrm{~d} n=5 ; 40 \mathrm{~d}$ $n=6$; foxo ${ }^{21} 1 \mathrm{wk} n=5$. Number of boutons quantified per sample analyzed: ywR $1 \mathrm{wk} n=177,25 \mathrm{~d} n=75,40 \mathrm{~d} n=96$, foxo ${ }^{21} 1 \mathrm{wk} n=100$. Number of branches analyzed: ywR $1 \mathrm{wk} n=39,25 \mathrm{~d} n=39,40 \mathrm{~d} n=40$, foxo ${ }^{21} 1 \mathrm{wk} n=39$.

TABLE 1 | Quantification of NMJ morphological characteristics of aging.

\begin{tabular}{|c|c|c|c|c|c|c|}
\hline & $\begin{array}{l}\text { Size of boutons } \\
\left(\mathrm{um}^{2}\right)\end{array}$ & $\begin{array}{l}\text { Branch length } \\
\text { (pixels) }\end{array}$ & $\begin{array}{c}\text { AZs/ } \\
\text { bouton }\end{array}$ & $\begin{array}{c}\text { AZs } \\
\text { /synaptic area } \\
\left(100 \mathrm{um}^{3}\right)\end{array}$ & Bouton number & Branch number \\
\hline ywR 1 wk & $3.16 \pm 1.84$ & $290.6 \pm 109.6$ & $4.07 \pm 0.51$ & $24.14 \pm 6.61$ & $90.5 \pm 18.41$ & $18.25 \pm 1.26$ \\
\hline$y w^{R} 25 d$ & $6.10 \pm 2.79$ & $219.5 \pm 55.28$ & $6.86 \pm 1.24$ & $23.06 \pm 8.22$ & $71.6 \pm 18.56$ & $14.5 \pm 0.71$ \\
\hline$y w^{R} 40 d$ & $7.91 \pm 4.23$ & $180.7 \pm 48.1$ & $7.81 \pm 0.95$ & $21.09 \pm 4.38$ & $52.0 \pm 11.86$ & $15.75 \pm 1.71$ \\
\hline foxo ${ }^{21} 1 \mathrm{wk}$ & $5.40 \pm 3.08$ & $238.2 \pm 69.66$ & $5.25 \pm 0.33$ & $21.54 \pm 3.825$ & $111.5 \pm 17.92$ & $28.33 \pm 7.57$ \\
\hline
\end{tabular}

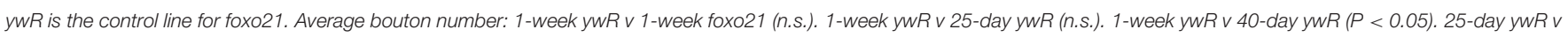

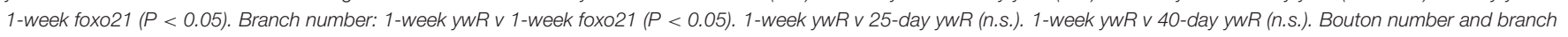
number represent both NMJs present at the A3 abdominal segment.

wild-type counterparts. However, the bouton area and branch length of foxo mutants was not significantly different from those seen in middle age control flies (Figures 1B,C, Table 1). The average active zone number between young wild-type and foxo mutants was not significantly different, but the foxonull mutants had more average AZs per bouton compared to controls (Figure 1D, Table 1, Supplementary Figure 1). When evaluating the raw total number of active zones per bouton, 
a significant difference was detected between 1-week controls and foxo mutants, but not between 25-day controls and 1-week foxo mutants (Supplementary Figure 1). Additionally, we did not detect a significant difference in overall AZs per synaptic area in foxo mutants when compared to the three measured control age groups. We did find that foxo mutants on average had more branches and a higher bouton number than wildtype flies (Table 1), which may be a compensatory response to reduced neurotransmission under excitatory conditions seen in foxo mutants (Howlett et al., 2008; Mahoney et al., 2016; McLaughlin and Broihier, 2018). We also examined bouton size and branch length in 1-week old flies containing a null foxo ${ }^{C 431}$ allele generated through CRISPR-Cas9 (Birnbaum et al., 2019). We found these null mutants also had enlarged boutons and shorter branch lengths compared to controls at a young age (Supplementary Figure 1). We validated that foxo protein levels were indeed reduced in our mutants (Supplementary Figure 1). Taken together, these results suggest that loss of foxo promotes aging-like morphological changes in boutons and synaptic branches at the adult neuromuscular junction.

\section{Loss of Foxo Results in Altered Cytoskeleton Structure in the Adult Neuromuscular Junction}

Axonal degeneration is a common consequence of aging and arises from a number of alterations to cellular and molecular pathways (Salvadores et al., 2017). This can result in a withdrawal

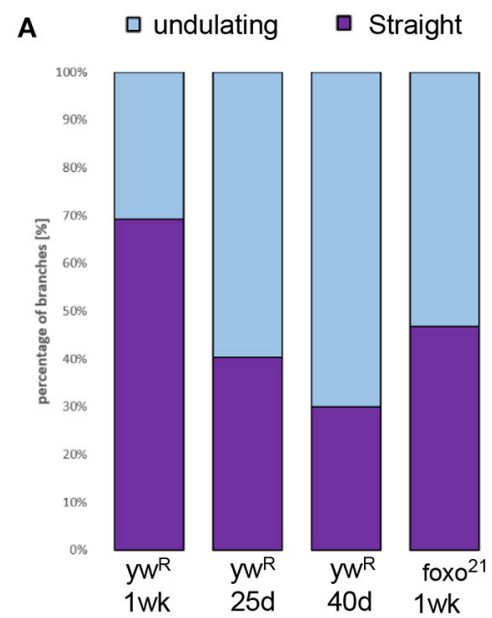

D
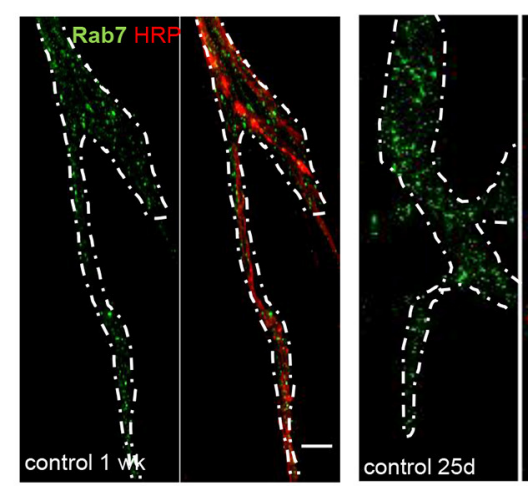

Bouton size 1wk
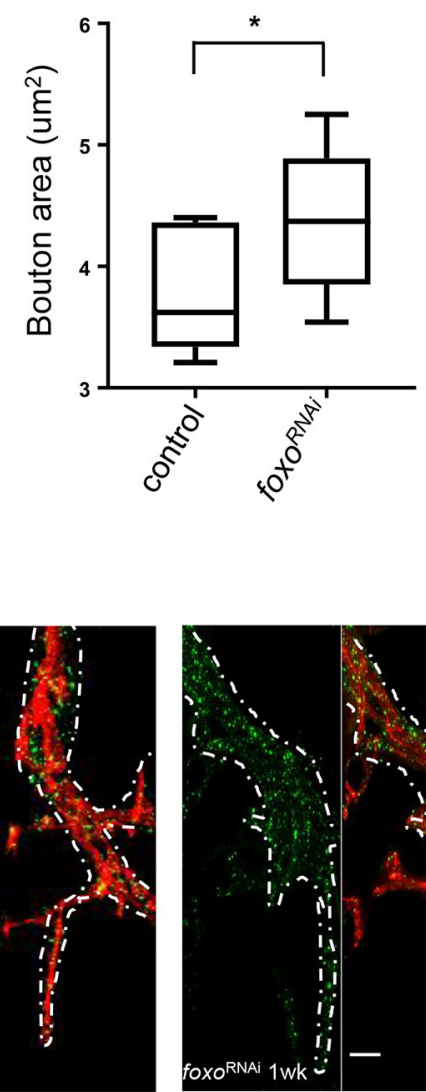

C
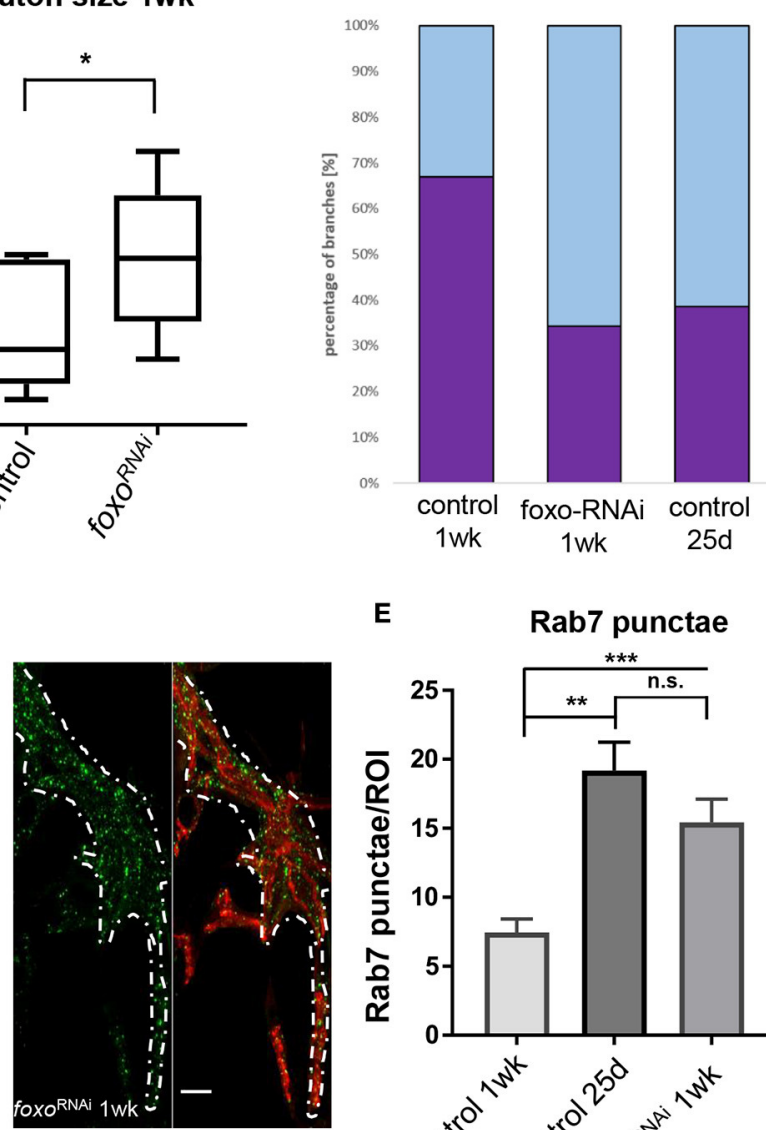

E

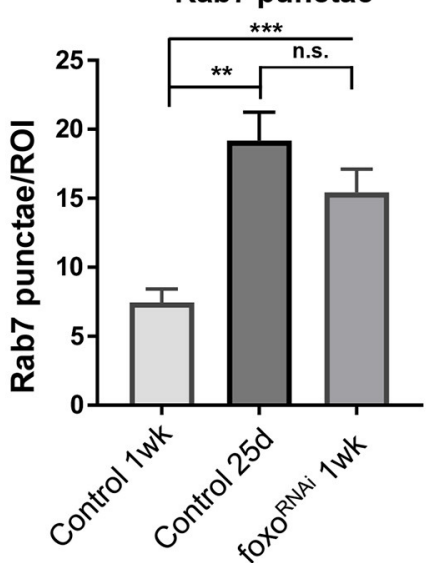

FIGURE 2 | Aging and loss of FOXO cause changes in microtubule structure and endocytic activity. (A) Quantification of anti-Ac-Tub at A3 VLM at control aging timepoints and in foxo mutant. The mean fraction of straight branches was significantly reduced from the young control for all other columns $(P<0.05)$. (B) Average bouton area for ok6-GAL4>yw ${ }^{R}$ compared to ok6-GAL4>foxo-RNAi \#1 ( $\left.{ }^{*} P<0.05\right)$. (C) Quantification of anti-Ac-Tub at A3 VLM at specified genotypes and ages. The $\%$ of straight branches of all other columns was significantly reduced from the young control $(P<0.05)$. (D) Representative images of main axon branchpoint with indicated genotypes and ages. Colabeled with Anti-Rab7 and anti-HRP. Nervous system tissue is outlined in white. Surrounding muscle tissue has been removed from the image. Bar $10 \mu \mathrm{M}$. (E) Number of Rab7 punctae detected per ROI in A3 segment. ${ }^{* \star} P<0.01$, ${ }^{\star \star \star} P<0.001$, n.s.- not significant. Number of animals analyzed for Ac-Tub: ywR 1 wk $n=3,25 \mathrm{~d} n=3,40 \mathrm{~d} n=3$, foxo ${ }^{21} 1 \mathrm{wk} n=3$, ok6-GAL4>yw 1 wk = 5, ok6-GAL4>yw $25 \mathrm{~d}=5$, ok6-GAL4> foxo-RNAi \#1 1

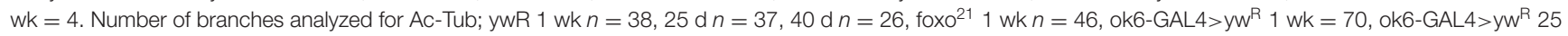
$\mathrm{d}=90$, ok6-GAL4> foxo-RNAi \#1 $1 \mathrm{wk}=75$. Number of animals analyzed for Rab7 quantification; ok6-GAL4>yw $1 \mathrm{wk}=7$, ok6-GAL4>yw $25 \mathrm{~d}=6$,

ok6-GAL4> foxo-RNAi \#1 $1 \mathrm{wk}=8$. 
of axons from their synaptic sites, caused by changes in the underlying cytoskeleton (He et al., 2002; Manini et al., 2013). Foxo proteins are known to regulate motor neuron microtubule dynamics during development across many species, and can cause a decrease in microtubule stability, allowing for increased plasticity (de la Torre-Ubieta et al., 2010; Nechipurenko and Broihier, 2012; McLaughlin et al., 2016). However, how foxo effects microtubule dynamics in a post-developmental model has not been examined. We used an antibody against acetylated alpha tubulin (Ac-tub) which marks stable microtubules at our three aging time points. We constructed a 3D model of each A3 segment and assigned all branches containing acetylated atubulin one of two characteristics; straight, or undulating. We quantified the percentage of each tubulin morphology found the undulating morphology significantly increases between 1week and 25 days, and between 25 and 40 days (Figure 2A, Supplementary Figure 2). In examining the wild-type flies, we found at the young time point around $70 \%$ of branches had a straight tubulin structure. As wild-type flies aged, we saw an increase in sinusoidal bends persisting throughout the branches, creating an undulating pattern (Supplementary Figure 2). This morphology can be associated with axonal retraction (He et al., 2002). These results show that there are changes that occur to the axonal microtubule structure with aging.

We next examined foxo mutants for any disruptions to the axonal microtubule morphology. Foxo mutants exhibited an increased number of undulating microtubules within branches and had significantly fewer straight microtubule bundles than the control at 1 week post eclosion (Supplementary Figure 2). When the 1-week foxo mutants were compared to the 25-day controls, there was no significant difference detected between the percentage of both undulating and straight microtubule branches (Figure 2A). To examine whether the change in microtubule morphology results from motor neuron specific foxo activity, we used a motor neuron specific Ok6-Gal4 to knock down foxo specifically in the motor neuron. We quantified bouton size between control and foxo-knockdown flies and found that knock-down of foxo in the motor neuron induces an increase in bouton size (Figure 2B). We stained A3 VLMs against Ac-tub, and at 1-week post eclosion, and observed nearly a 2 -fold decrease in the number of straight branches in knockdown flies compared to controls (Ok6-Gal4>ywR) (Figure 2C, Supplementary Figure 2). We also compared our 1-week foxo knockdown to 25-day controls and found there to be no significant difference between the percentage of undulating and straight microtubules within axonal branches (Figure 2C, Supplementary Figure 2). Thus, these findings suggest that foxo regulates bouton size and axonal microtubule morphology autonomously.

\section{Loss of Foxo Causes Alteration to the Endocytic Pathway in the Adult Neuromuscular Junction}

Aging has been shown have disrupted endosome-lysosome trafficking and increased numbers of early endosomes and multivesicular bodies (prerequisites for late endosomes) at the synapse, which can result in neurodegeneration (Boaro et al., 1998; Nixon et al., 2008; Wagner et al., 2015; Colacurcio and Nixon, 2016). In C. elegans, late endosome formation is suppressed in organisms with delayed aging (Richardson et al., 2019). Additionally, late endosomes undergo retrograde transport to degrade cargo, and this transport declines with age (Milde et al., 2015; De Vos and Hafezparast, 2017). To observe changes in the endocytic degradation pathway, we used an antibody against Rab7 to mark late endosomes. We performed immunofluorescent staining at the A3 VLM on 1-week and 25day adults. To our surprise, we observed Rab7-marked punctae around the axon branchpoint site and noticed a visible increase at this site in middle-aged flies. To quantify the number of Rab7 vesicles, we generated circular regions of interest (ROIs) of the same size and used HRP to identify z-slices containing the axon. The slice with the highest punctae number for each ROI was collected and averaged for each specimen. We found the 25-day adult flies had more than 2-fold more Rab7 punctae per ROI associated with the axon branch than the 1-week adults, demonstrating there is an increase in the number of late endosomes during aging (Figures 2D,E).

We next examined the effects of motor neuron specific knockdown of foxo in late endosome signaling. We found that foxo KD flies had significantly more punctae per ROI than the young controls in nervous system tissue (Figures 2D,E, Supplementary Figure 4). We tested a second foxo-RNAi line (foxo-RNAi \#2) and again observed significantly more Rab7 punctae at the branchpoint than controls (Supplementary Figure 3). To test whether the morphological changes of foxo knockdown flies are due to the developmental defects, we examined for Rab7 accumulation, bouton size and ac-tubulin structure from the female flies 2 day poste eclosion. We found no significant differences were detected between control and foxo-RNAi flies (Supplementary Figure 4). Overall, both aging and foxo knockdown result in an increase in the number of late endosomes around the branchpoint of motor neuron, suggesting a potential role of foxo in age-related alterations in endocytic pathway.

\section{Overexpression of foxo Rescues NMJ Aging Phenotypes}

To test if overexpression of foxo can restore age-related NMJ defects, we crossed Ok6-GAL4 to UAS-foxo flies to express the wild-type foxo transgene specifically in the motor neuron. At 1-week post eclosion, no differences were detected in Rab7 number between controls and foxo overexpression flies (Figures 3A-C). At 25 days, foxo overexpression flies exhibited significantly fewer late endosomes than control flies (Figures 3A,B,C). Consistently, foxo overexpression attenuated age-related increases in bouton size (Figure 3D). To test for the effectiveness of foxo overexpression, we measured the expression of a known foxo target gene, Thor (4EBP) (Junger et al., 2003). We found a 2 -fold increase in Thor expression in foxo overexpression flies (Supplementary Figure 3). Taken together, these results indicate that overexpression of foxo can preserve motor neuron morphology and endocytic activities. 

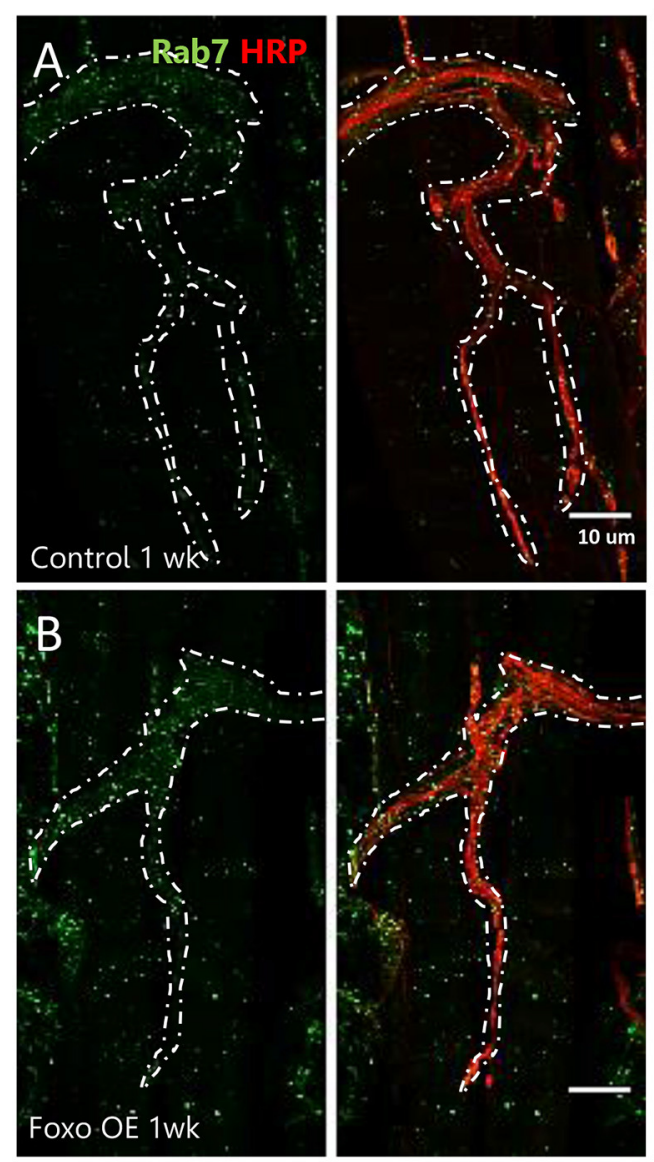

C
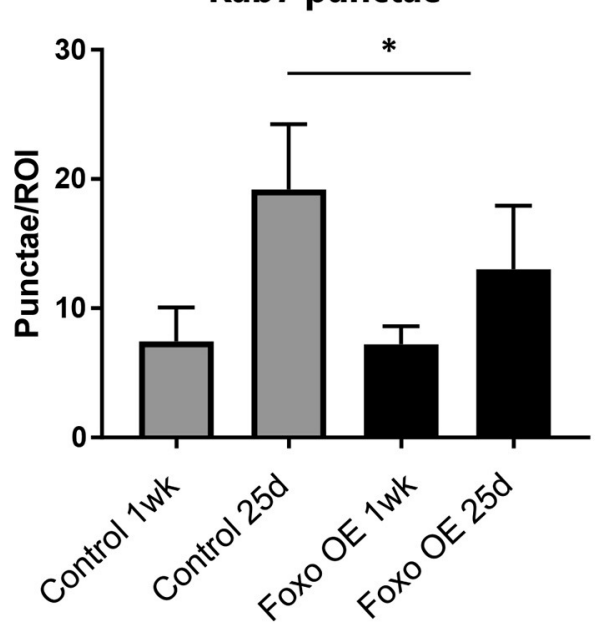
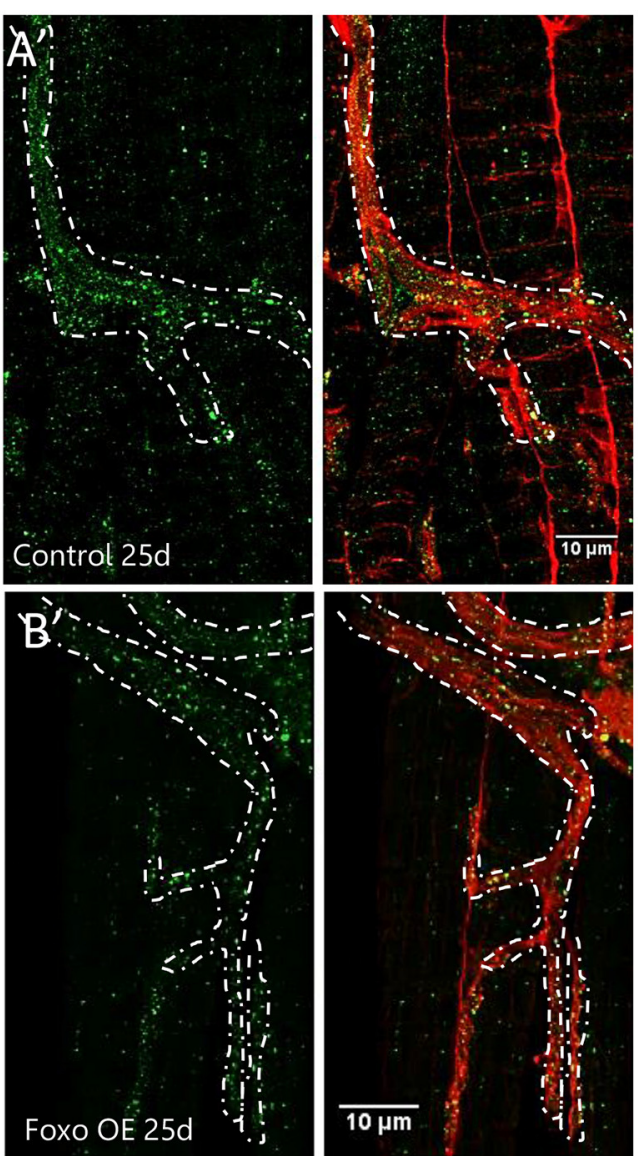

D

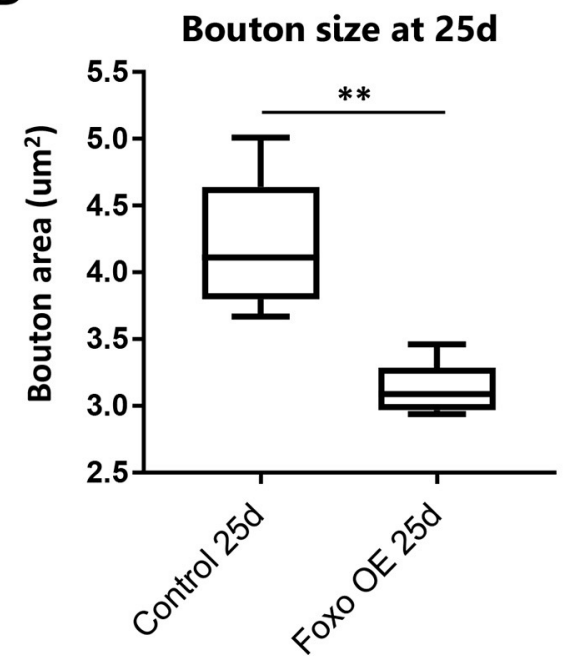

FIGURE 3 | Overexpression of motor neuron FOXO delays aging phenotypes (A,B). Representative figures of control and FOXO-overexpression at 1-week, (A',B') and 25 days post eclosion. Colabelling with anti-Rab7 and anti-HRP. Scale bar $10 \mu \mathrm{m}$. (C) Rab7 quantification for A3 ROls for indicated genotypes and ages ${ }^{\star} P<$ 0.05. (D) Average bouton area for 25-day old FOXO overexpression and control ${ }^{\star \star} P<0.01$. Number of animals analyzed for Rab7 quantification; ok6-GAL4>yw ${ }^{R} 1$ $w k=7$, ok6-GAL4>yw $25 d=6$, ok6-GAL4> UAS-foxo 1 wk $=4$. ok6-GAL4> UAS-foxo $25 d=7$. Number of animals analyzed for bouton size: ok6-GAL4>yw ${ }^{R}$ $25 \mathrm{~d}=5$, ok6-GAL4 $>$ UAS-foxo $25 \mathrm{~d}=5$. 


\section{MAPK and Activin Signaling Genetically Interact With Foxo to Control NMJ Homeostasis}

In order to dissect the mechanisms underlying foxo-regulated NMJ homeostasis, we conducted a genetic screening to uncover the downstream targets of foxo in the motor neuron. The candidate genes were selected from our previous foxo ChIPSeq and transcriptome analyses (Birnbaum et al., 2019). We selected 207 candidate genes with positive foxo binding (fold change $>1.5$ ) and differential expression (fold change $>1.25$, $p<0.05)$ (Figure 4A, Supplementary Table). Interestingly, these candidate genes were enriched for biological processes involving neurotransmitter secretion, cytoskeletal organization, and signaling pathways such as MAPK (Supplementary Table).

Among all identified candidate genes, we prioritized 15 candidates in the genetic screening. Rab7 and bouton morphology were used as NMJ readouts (Table 2). We first generated a fly line by combining Ok6-gal4 driver and UAS-foxo$R N A i$. Through the screening, we identified four genes whose knockdown attenuated the accumulation of late endosome number in foxo KD flies. We also found that overexpression of two genes Thor and Ank2 significantly reduced Rab7 number of the foxo KD flies. Among identified target genes, three genes, JNK transcription factor kay (Kayak), type I activin receptor babo (baboon), and p38b MAP kinase (p38b), are known to have regulatory effects on axonal transport (Ellis et al., 2010; Drerup and Nechiporuk, 2013; Gibbs et al., 2018). Similarly, knockdown of five candidate genes (lqf, babo, kay, p38b, grk) blocked the induction of bouton size in foxo $\mathrm{KD}$ flies (Figure 4C). Thus, our findings indicate that TGF- $\beta$ and MAPK signaling are the downstream effectors that mediate foxo-regulated NMJ homeostasis.

We further confirmed that foxo knockdown significantly increased the phosphorylation of p38 in the motor neuron (Figures 5A,B), suggesting that foxo might suppress MAPK signaling in the motor neuron.

\section{DISCUSSION}

Foxo is known to act a regulator of synaptic plasticity and microtubule stability (Howlett et al., 2008; Nechipurenko and Broihier, 2012). Although we have seen foxo's regulation at the NMJ during development, it is unclear the role it plays in NMJ maintenance during aging. Foxo promotes youthful characteristics in many different tissues and has been shown to delay the onset of degenerative properties (Salih and Brunet, 2008; Demontis and Perrimon, 2010). Here we demonstrate that foxo is a regulator of NMJ homeostasis in adult motor neurons. Our data suggests that foxo plays a functional role in maintaining the motor neuron during adulthood and can maintain youthful neuronal phenotypes post-development. We have found that motor neuron expressed foxo contributes to the morphology of synaptic contacts and can regulate cytoskeletal and endocytic processes within neuronal tissue. Our results also suggest that TGF- $\beta$ and $\mathrm{p} 38$ /MAPK signaling function downstream of foxo to control these processes. The changes in foxo behavior during aging may induce p38 activation, resulting in disruption of homeostatic mechanisms and promoting cellular senescence.

In the present study, we show that loss of foxo causes morphological changes to synaptic structures in young adult female drosophila. While foxo mutants have been previously shown to have larger boutons compared to controls during development, they were not reported to have any changes in branch length or number, nor were any differences reported in bouton number (Nechipurenko and Broihier, 2012; McLaughlin et al., 2016). We have found this not to be the case in the adult. Our results showed that at 1-week post eclosion, foxo mutant flies had shorter branches compared to control flies. They also had a significantly larger number of branches, which ultimately resulted in a larger bouton number per muscle tissue. We hypothesize that this increase in branching may be due to a compensatory response, as foxo mutants have been shown to have reduced neurotransmission (Howlett et al., 2008; Mahoney et al., 2016), which may cause a need for more synaptic contacts. Additionally, foxo mutants are shown to have increased microtubule stability due to reduced microtubule sliding (McLaughlin et al., 2016). This may inhibit growth to postsynaptic targets, resulting shorter branches. The increase in branch number is contrary to what is observed in Drosophila larval dendrites, where loss of foxo reduces arborization (Sears and Broihier, 2016). foxo has also been shown to reduce neurite branching in aged C. elegans downstream of both JNK and Insulin signaling (Tank et al., 2011). However, increases in ROS can induce overgrowth at the Drosophila NMJ, and foxo is known to mitigate oxidative stress through antioxidant transcription activation (Essers et al., 2004; Milton et al., 2011). This increase may also be the result of ineffective pruning during metamorphosis.

Active zone number is proposed to proportionally increase with bouton size under a healthy aging model (Wagner et al., 2015). Although there was no significant difference between average $\mathrm{AZ}$ number between controls and foxo mutants, we did observe more AZ's per bouton in the mutants. Previous studies have shown that accumulation of Brp in axons can limit active zone number at synaptic terminals (Barber et al., 2018). Foxo mutant larvae have been shown to have accumulations of Brp in the main axon (McLaughlin et al., 2016), but we were unable to capture this in the adult. Based on our current data, we cannot determine whether foxo influences active zone number. Further experimentation using Electron Microscopy of t-bars in presynaptic area could answer this question.

Aging neurons undergo cytoskeletal changes in order to maintain plasticity and synaptic contact to muscle tissue (Mattson and Magnus, 2006). As previously mentioned, foxo has been shown to regulate the axonal cytoskeleton and neuronal plasticity. During development, foxo mutant larvae have increased futch positive looping and acetylated alpha tubulin is present in the terminal boutons (Nechipurenko and Broihier, 2012). Boutons were also shown to have altered AcTub bundling patterns compared to controls (McLaughlin et al., 2016). In our adult flies, we observed an increase in sinusoidal Ac-Tub morphologies among presynaptic branches, and this 
A

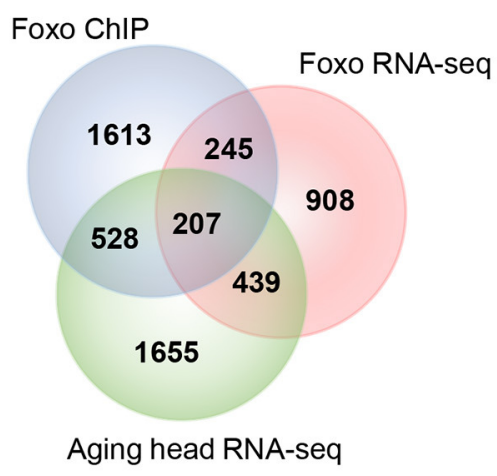

C

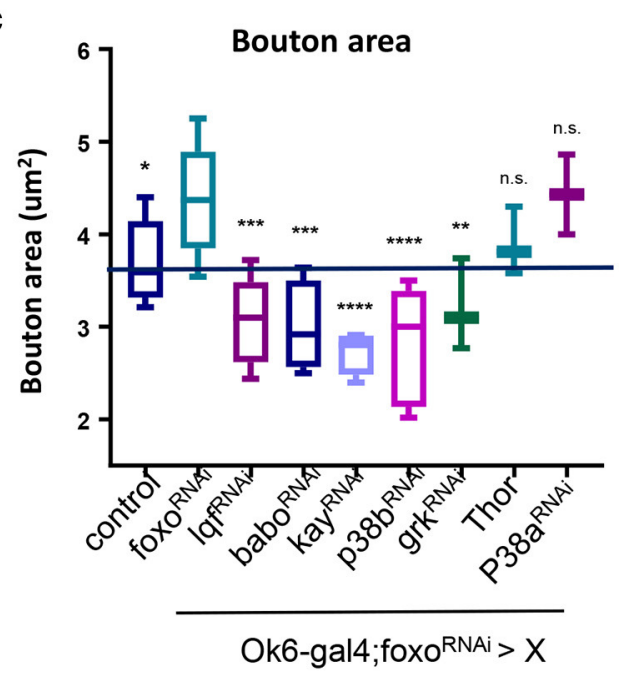

B

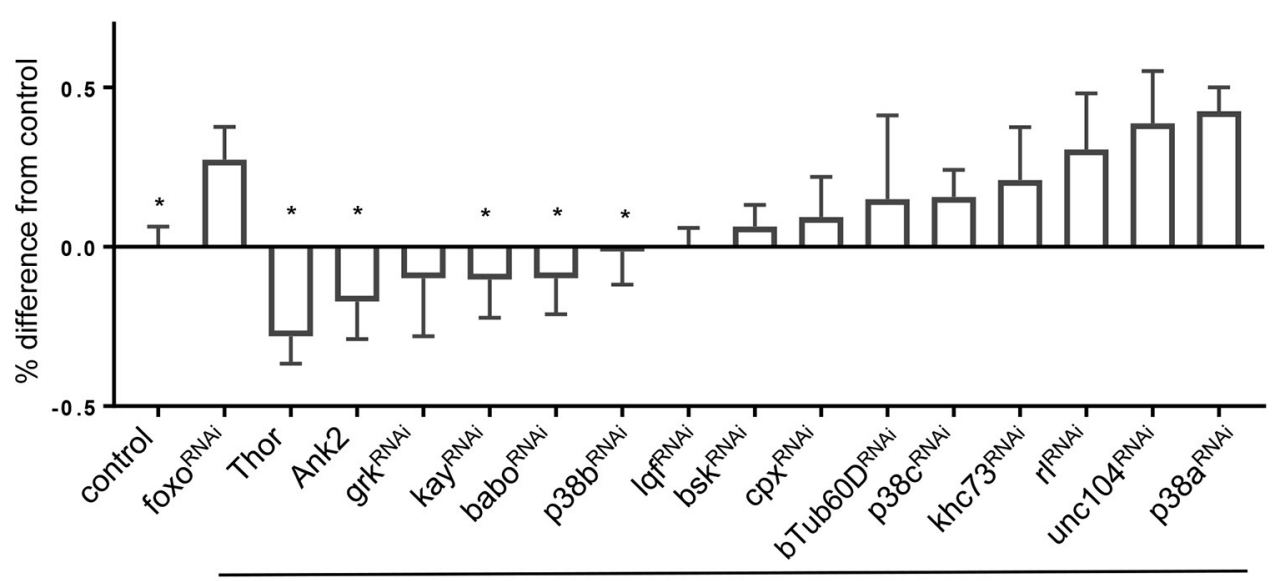

Ok6-gal4;foxoRNAi > X

FIGURE 4 | MAPK acts downstream of FOXO to regulate NMJ pathways. (A) Venn diagram overlap of ChIP-seq, head foxo ${ }^{\text {C431 }}$ RNA-seq, and aged head RNA-seq: For RNA-seq data $\{-0.15>\log 2 \mathrm{FC}>0.15, P<0.05\}$. \{FOXO ChIP FC $>1.5$, FDR $<0.05\}$. (B) Quantification of Rab7 for double mutant flies. Values are set as a percentage with ok6>yw ${ }^{R}$ as baseline. All significant values are compared to foxo ${ }^{\mathrm{RNAi}}$ (in ok6-GAL4 background) ${ }^{*} P<0.05$. (C) Average bouton size quantification for indicated genotypes. Control in Ok6-GAL4 background. ${ }^{\star} P<0.05,{ }^{\star \star} P<0.01,{ }^{\star \star \star} P<0.001$, ${ }^{\star \star \star \star} P<0.0001$, n.s., not significant. Number of animals used for (B) in order from left to right: $n=22 ; 13 ; 7 ; 4 ; 3 ; 6 ; 10 ; 7 ; 4 ; 6 ; 5 ; 4 ; 6 ; 6 ; 6 ; 3 ; 3$. Number of animals used for (C) bouton size in order from left to right: $n=14 ; 11 ; 4 ; 5 ; 4 ; 5$; 3; 3; 2. Number of animals used for (B) c-Tub: ok6-GAL4; foxo-RNAi >ywR = 4, ok6-GAL4; foxo-RNAi > babo-RNAi =6, ok6-GAL4; foxo-RNAi > p38b-RNAi = 5. Number of branches used for (B) c-Tub: ok6-GAL4; foxo-RNAi >ywR = 75, ok6-GAL4; foxo-RNAi> babo-RNAi = 93, ok6-GAL4; foxo-RNAi > p38b-RNAi = 80.

is likely a contributing factor to the truncation of established branches during aging. Dynamic stretching of axons leads to undulating distortions and mechanical failure along the axon (Tang-Schomer et al., 2010). These events have been well-studied in injury models, but less so in aging, where regeneration of axonal damage becomes altered (Kleele et al., 2014; Hill et al., 2016; Geoffroy et al., 2017). Additionally, inhibition of dynein has been shown to cause bending in microtubules (Kent et al., 2016). Splayed branching in foxo mutants and motor neuron knockdown flies may be an artifact from the altered bundling pattern observed in the larvae (McLaughlin et al., 2016). While the cause of this is unknown, all microtubule strands appear to be in the same phase of action, in that they are either all straight or all undulating. Additionally, in developing fly larvae, a constitutively active form of foxo caused severe microtubule destabilization, while overexpression of wild-type foxo did not have the same disruption (Nechipurenko and Broihier, 2012). This may indicate that foxo nuclear activity is not solely responsible for maintaining motor neuron integrity.

Endocytosis and exocytosis play important roles in maintaining both the pre- and postsynaptic regions. Endosometo-lysosome trafficking is disrupted in aging motor neurons, and multivesicular bodies, which are prerequisites for late endosomes, have been shown to accumulate in the presynaptic space during aging (Forester et al., 2010; Wagner et al., 2015). Additionally, disruption of this shuttling is a known symptom of neurodegenerative diseases (Takahashi et al., 2002). In our foxo knockdown flies, we found an increase in the number 
TABLE 2 | Downstream candidate genes from FOXO ChIP-seq and RNA-seq.

\begin{tabular}{|c|c|c|c|c|c|}
\hline Gene symbol & Gene name & Function & ChIP FC & FOXO RNA-seq log2FC & Aging head RNA-seq log2FC \\
\hline $\mathrm{rl}$ & Rolled & ERK & 4.73 & 0.343 & 0.159 \\
\hline Lqf & Liquid facets & Epsin1 & 2.67 & 0.386 & 1.132 \\
\hline Unc-104 & Uncoordinated-104 & Kinesin/KIF1A & 2.33 & -0.388 & 0.327 \\
\hline Khc-73 & Kinesin heavy chain 73 & Kinesin & 1.94 & -0.23 & 0.106 \\
\hline Ank2 & Ankyrin 2 & Microtubule associated protein & 3.48 & -0.433 & 0.468 \\
\hline Cpx & Complexin & SNARE regulator & 3.68 & -0.22 & 1.001 \\
\hline bTub60D & Beta-Tubulin at 60D & Microtubule building block & 1.91 & 1.442 & -1.147 \\
\hline Babo & Baboon & Activin receptor & 3.71 & 0.244 & 0.258 \\
\hline Grk & Gurken & ERK ligand & 2.61 & 0.292 & -0.560 \\
\hline Thor & Thor & 4EBP & 3.24 & -0.225 & -0.137 \\
\hline Bsk & Basket & JNK & - & -0.343 & 0.529 \\
\hline Kay & Kayak & JNK Transcription factor & 2.55 & -0.059 & -0.302 \\
\hline P38a & p38a MAP kinase & P38 kinase & - & 0.211 & -0.737 \\
\hline P38b & p38b MAP kinase & P38 kinase & - & 0.133 & 0.358 \\
\hline p38c & p38c MAP kinase & P38 kinase & - & -0.305 & -0.946 \\
\hline
\end{tabular}

FC, Fold change.
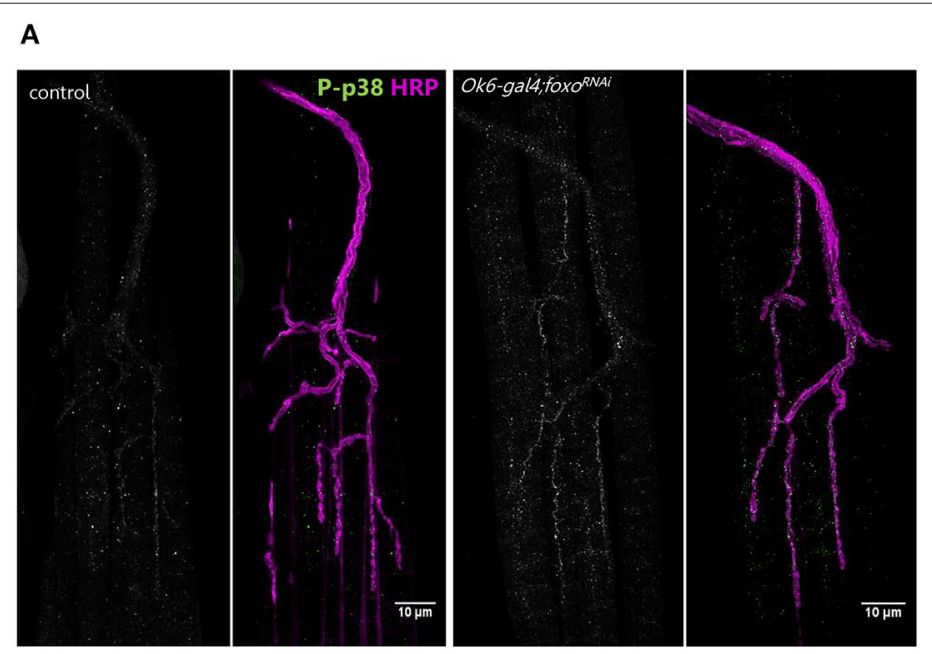

\section{B}

Phosphorylated p38

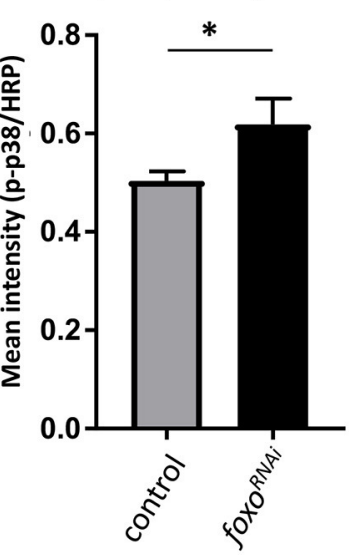

FIGURE 5 | Motor neuron FOXO inhibits MAPK activation. (A) Representative images of 1-week old fly NMJs. Colabeled with anti-phosphorylated-p38 and anti-HRP. Scale bar $10 \mu \mathrm{m}$. (B) Relative intensity of P-p38 at anti-HRP masked areas. Intensity was normalized to anti-HRP intensity. ${ }^{*} P<0.05$. Number of NMJs used: ok6-GAL4 >ywR 1wk = 9; ok6-GAL4; foxo-RNAi >ywR 1 wk = 10 .

of late endosomes associated with the axon. This was also observed with aging. Late endosomes are involved for the shuttling of damaged cargo to autophagosomes and lysosomes for degadation, which may indicate an increase in autophagy as a protection mechanism against harmful stress (Maiuri and Kroemer, 2015; Kaur and Lakkaraju, 2018). It has been shown that vesicle axonal transport declines with age, as does lysosome acidification (Forester et al., 2010; Milde et al., 2015; Vagnoni and Bullock, 2018). Although foxo has been shown to promote Rab7 protein expression to promote autophagy in cardiomyocytes, this was in a glucose restricted model and suggests a different mechanism than what we are observing with age (Hariharan et al., 2010). Apoptotic neurons can activate glial innate immunity mechanisms, which involved upregulation of endocytic vesicles in glia to eliminate debris. foxo null-mutants have increased apoptotic debris in the brain during development, and loss of foxo expression in larval cortical glia has reduced phagocytosis and enhanced neurodegeneration (McLaughlin et al., 2019). It is therefore possible that loss of foxo in the motor neuron results in the exportation of damaged cargo to the glia for elimination. However, more experimentation needs to be done to determine the location and underlying causes for this increase in late endosomes. Meanwhile, overexpression of foxo delays late endosome increase in our adult flies.

Through our epistasis analysis, we found knocking down members of MAPK and TGF $\beta$ signaling have a profound rescue effect on the late endosome accumulation seen in foxo-RNAi flies. We observed that knockdown of the activin receptor babo 
was able to rescue late endosome accumulation. TGF $\beta$ signaling has been shown to regulate axon development and Rho GTPase activity in mushroom bodies and dictates postsynaptic density abundance at the NMJ through motor neuron anterograde signaling (Ng, 2008; Kim and O'Connor, 2014). TGF $\beta$ signaling has also been shown to promote aging in cardiac tissue (Chang et al., 2019). We also observed that the MAP kinase p38b was able to rescue late endosome upregulation near the control baseline. In ALS mouse models, inhibition of p38 is able to rescue axonal retrograde trafficking (Gibbs et al., 2018), and p38b is specifically shown to regulate synaptic morphology in drosophila, though in response to stress both $\mathrm{p} 38 \mathrm{a}$ and $\mathrm{p} 38 \mathrm{~b}$ are show to act redundantly in Drosophila, mammals, and yeast (AdachiYamada et al., 1999; Klinedinst et al., 2013). Overexpression of MAPK has been shown to increase larval bouton number, and p38 gain of function results in increased oxidative stress, a known target of foxo activity (Koh et al., 2002; Zhan et al., 2015). In mammals, activation of $\mathrm{p} 38 / \mathrm{MAPK}$ is associated with increased neuroinflammation, neurodegenerative disease progression, and disruption of synaptic function (Correa and Eales, 2012; Falcicchia et al., 2020). Additionally, TGF $\beta$ has been shown to activate p38 independently of SMAD and can induce cell apoptosis through this pathway (Yu et al., 2002). p38 activation has also been associated with increased ROS formation and the promotion of pro-inflammatory cytokines (Zhao et al., 2018). In neuronal tissue, increased ROS and inflammation result in the activation of microglia, with chronic activation being able to induce neuronal damage and apoptosis (Dheen et al., 2007; Gwak et al., 2017). p38 signaling has been shown to regulate Rab7 expression in response to interleukin cytokines (Bhattacharya et al., 2006), further supporting that the increase in Rab7 observed during aging and under foxo knockdown may be a response to increased inflammation.

In our screen we also targeted rolled $(r l)$, which is the Drosophila ortholog of ERK1/2 (Biggs et al., 1994). We expected to see a decrease in late endosome accumulation but were surprised to find there was no change. We hypothesize that this is due to the potential for two forms of ERK signaling. These two mechanisms of ERK signaling may control opposing processes of synaptic transmission. Rolled hypomorphs show a reduced excitatory junction potential, similar to what is seen in foxo hypomorphs, suggesting reduction of rolled plays a part in synaptic transmission that may be independent of its role in late endosome shuttling (Wairkar et al., 2009). Additionally, blockage of endosome-lysosome fusion will lead to an increase of activated ERK (Ng and Tang, 2008), suggesting it may still have functional consequences downstream of foxo activity.

In summary, we have found that loss of foxo in the adult Drosophila results in morphological changes to synaptic structure that resemble aging morphologies. We also show that motor neuron foxo is required for endocytic homeostasis, and enhanced foxo expression during adulthood can delay aging morphologies. We find evidence to support that foxo may act as a repressor of TGF $\beta$ and MAPK signaling, particularly p38 signaling, to maintain youthful characteristics at the NMJ. Identifying the cellular mechanisms regulated by these pathways will provide a greater understanding into how synaptic plasticity is maintained and what homeostatic processes preserve synaptic function during aging.

\section{METHODS}

\section{Fly Stocks and Husbandry}

The following stocks were used: $y w R$ (Rochele), $y w R ;+: f o x o^{21}$, $w^{1118}$, foxoC431 (Gift from N. Liu, Chinese Academy of Sciences, Shanghai, China), Ok6Gal4 (Gift of E McNeill, Iowa State University), mhcGal4 (Demontis and Perrimon, 2010, Cell). Flies were collected every $24 \mathrm{~h}$, mated than sorted 2-3 days after eclosion. Flies were placed in vials containing standard CSY food. Fly strains were maintained at $25^{\circ} \mathrm{C}$ with 12 -h light/dark cycle, and $60 \%$ humidity. Flies were transferred to fresh food every 3 days. Overexpression and TRiP RNAi lines were obtained from the Bloomington stock center unless otherwise specified: UAS-foxo (BDSC_42221), foxo-RNAi \#1 (BDSC_32993), foxo-RNAi \#2 (BDSC_32427), ATTP40 (y sc v; attP40 (genotype: $y[1] \mathrm{sc}[1] \mathrm{v}[1] ; \mathrm{P}\{\mathrm{y}[+\mathrm{t} 7.7]=\mathrm{CaryP}\}$ attP40)), rl-RNAi (BDSC_34855), khc-73-RNAi (BDSC_36733), lqf-RNAi (BDSC_58130), cpx-RNAi (BDSC_42017), unc-104-RNAi (BDSC_58191), UAS-Ank2GFP (Gift from Ronald Dubreuil, University of Illinois at Chicago), Bsk-RNAi (BDSC_57035), kay-RNAi (BDSC_33379), babo-RNAi (BDSC_25933), p38aRNAi (BDSC_34744), p38b-RNAi (BDSC_29405), p38c-RNAi (BDSC_64846), grk-RNAi (BDSC_55926), bTub60D-RNAi (BDSC_64856), UAS-Thor (BDSC_9147. Fly lines $\mathrm{w}^{1118}$;ok6Gal4 and BL32993 (foxo-RNAi) were crossed to double balancer yw;Sp/Cyo;TM2e/TM6BTbHue (Rochele). Markers were used to select carriers. After Ok6 was combined with the foxo-RNAi, lines were backcrossed for 5 generations before use. Flies containing Ok6 in a yw background were self-crossed to produce a control strain. Line was backcrossed for 5 generations. A daughterless gene switch GAL4 driver was used to validate foxo knockdown and overexpression.

\section{Immunofluorescence Staining}

Flies were subjected to flynap (Carolina, Burlington, NC) and dissected to expose the ventral abdominal muscles [protocol from Wagner et al. (2015)] in $\mathrm{Ca}^{2+}$ free saline $[128 \mathrm{mM}$ $\mathrm{NaCl}, 2 \mathrm{mM} \mathrm{KCl}, 4 \mathrm{mM} \mathrm{MgCl}{ }_{2}\left(\mathrm{H}_{2} \mathrm{O}\right)_{6}, 35.5 \mathrm{mM}$ sucrose, $5 \mathrm{mM}$ HEPES, $1 \mathrm{mM}$ EGTA, $\mathrm{H}_{2} \mathrm{O}, \mathrm{pH}$ 7.2]. Tissue was then fixed in $4 \%$ paraformaldehyde for $20 \mathrm{~min}$ at room temperature. Tissue was washed in $1 \mathrm{X}$ PBST $(0.1 \%$ Triton $\mathrm{X})$ and blocked with $5 \%$ NGS (normal goat serum) for $1 \mathrm{~h}$ at room temperature. Samples we incubated overnight at $4^{\circ} \mathrm{C}$ with 2 ug primary antibodies in 5\% NGS 1X PBST [1X phosphate buffer saline, 0.1\% Triton X-100]. Primary antibodies used were as follows: antiBRP (DSHB nc82), anti-Dlg (DSHB 4F3), anti-Rab7 (DSHB), anti-Rab7 (gift from Nakamura lab), anti-Acetlyated Tubulin (Sigma Aldrich T7451), anti-phospho-p38 MAPK (CST 9211). Tissues we washed in 1XPBST and incubated $1.5 \mathrm{~h}$ at room temperature in 1XPBST and anti-HRP-594 or HRP-647 (Jackson ImmunoResearch Laboratories Inc, West Grove PA) at a Dilution of 1:200 with secondary antibodies (1:250) and kept in the dark at room temperature for $2 \mathrm{~h}$. Samples were washed in PBST and mounted in Prolong Diamond (Life technologies). For Fat body 
analysis, pelts were incubated in slowfade gold with DAPI (life technologies) at $4^{\circ} \mathrm{C}$ overnight prior to mounting.

\section{Secondary Antibodies}

All secondary antibodies used were from Jackson ImmunoResearch Laboratories Inc and were as follows: 488 donkey anti-mouse, 488 goat anti-rabbit, rabbit anti-HRP-594, donkey anti-HRP-647.

\section{Imaging Analysis and Quantification}

Images were captured using an epifluorescence-equipped BX51WI microscope (Olympus, Waltham, MA, USA) and Olympus FV3000 laser scanning confocal (Olympus, Waltham, MA, USA). CellSens software (Olympus, Waltham, MA, USA) was used for deconvolution of stacks. For aging metrics quantifications, maximum $\mathrm{Z}$ projections were generated of whole A3 muscle segment. Bouton size and active zone number were quantified in CellSense through a selected region of interest (ROI) aligned to each bouton. Measurements were performed using the "measure and count" module, providing quantification of bouton and active zone number and area. Branch number and branch length were quantified in Fiji (imageJ, Schindelin et al., 2012). Acetylated alpha-Tubulin quantification was performed via manual counting using the volume module in Fluoview software. Rab7 quantification was performed in CellSense using a circle we generated a circular ROI (25.75 $\mu \mathrm{m}^{2}$ ) to quantify number of punctae. Z-slices quantified were limited to those containing HRP staining at selected regions to avoid non-neuronal associated punctae. ROIs were checked prior to quantification to ensure muscle associated punctae were removed. Intensities for pp38, pERK, and pJNK were conducted using Fiji (NIH) by measuring the sum fluorescence intensity in ROIs defined by HRP immunoreactivity. HRP fluorescence intensity was measured to ensure no differences between control and mutant NMJs. All images were generated using Fiji.

\section{Quantitative Real-Time PCR}

Three-day post eclosion flies were placed on food containing $<0.1 \%$ ethanol and $200 \mathrm{uM}$ Mifepristone (Cayman Chemical) or no other additive for 3 days. Whole bodies of female flies were homogenized in TRIzol (Thermo Fisher). RNA was extracted followi ng company procedure. cDNA was generated using qScript (Quanta) and diluted to a $10 \mathrm{uM}$ working solution. Quantitative PCR was run on purified samples (QuantStudio). Sybrrgreen (Life Technologies, CA, USA) was used for chemical detection. The primers used Enrichment was determined based on the double-delta CT value. Thor (sense, $5^{\prime}$-CCAGGAAGGTTGTCATCTCGG-3', and antisense $5^{\prime}$ - CTCGTAGATAAGTTTGGTGCCTCC-3'), Rpl32 (sense 5' AAGAAGCGCACCAAGCACTTCATC- $3^{\prime}$, and antisense $5^{\prime}$ TCTGTTGTCGATACCCTTGGGCTT-3').

\section{Video Capturing}

Stacks of the entire A3 muscle region were acquired with Olympus FV3000 laser scanning confocal in the Fluoview software (Olympus, Waltham, MA, USA). Videos were recorded of $3 \mathrm{D}$ renderings using the movie module under the volume setting.

\section{Venn Diagrams}

Venn comparisons were performed using the Bioinformatics and Evolutionary Genomics Venn calculator at http://bioinformatics. psb.ugent.be/webtools/Venn/.

\section{Statistical Analysis}

GraphPad Prism (GraphPad Software) was used for statistical analysis. To compare the mean value of treatment groups vs. that of control, either student $t$-test or one-way ANOVA was performed using Tukey multiple comparison. All genetic screen lines were compared to the generated foxo-RNAi line using a student $t$-test. The metrics during aging were analyzed by twoway ANOVA, including Tukey multiple comparisons test. Before analysis, outliers were identified using Robust regression and Outlier removal (ROUT) method $(\mathrm{Q}=1 \%)$.

\section{DATA AVAILABILITY STATEMENT}

The raw data supporting the conclusions of this article will be made available by the authors, without undue reservation.

\section{AUTHOR CONTRIBUTIONS}

$\mathrm{AB}, \mathrm{MS}, \mathrm{MB}, \mathrm{KC}$, and $\mathrm{PK}$ : data acquisition. $\mathrm{AB}, \mathrm{EM}$, and $\mathrm{HB}$ : design, analysis, interpretation, and manuscript revision. $\mathrm{AB}$ and $\mathrm{HB}$ : conception. AB: manuscript writing. $\mathrm{HB}$ : resources and funding acquisition.

\section{FUNDING}

This work was supported by NIH/NIA R00 AG048016 to HB, AFAR Research Grants for Junior Faculty to HB.

\section{ACKNOWLEDGMENTS}

We thank Bloomington Drosophila Stock Center (BDSC) and Norbert Perrimon and others at the Drosophila RNAi Screening Center (DRSC) for providing fly lines. We also thank Nan Liu and Xiaofen Wu (Chinese Academy of Sciences, Shanghai, China) for fly lines and RNA-seq data, and Ronald Dubreuil (University of Illinois at Chicago, Chicago, IL) for fly lines used in this study. We thank Akira Nakamura (RIKEN Center for Developmental Biology, Kobe, Japan) for antibodies used in this study. The authors acknowledge the assistance of rotation student Sean McLaughin.

\section{SUPPLEMENTARY MATERIAL}

The Supplementary Material for this article can be found online at: https://www.frontiersin.org/articles/10.3389/fnagi. 2020.567861/full\#supplementary-material 


\section{REFERENCES}

Accili, D., and Arden, K. C. (2004). Foxos at the crossroads of cellular metabolism, differentiation, and transformation. Cell 117, 421-426. doi: 10.1016/s0092-8674(04)00452-0

Adachi-Yamada, T., Nakamura, M., Irie, K., Tomoyasu, Y., Sano, Y., Mori, E., et al. (1999). p38 mitogen-activated protein kinase can be involved in transforming growth factor beta superfamily signal transduction in Drosophila wing morphogenesis. Mol. Cell Biol. 19, 2322-2329. doi: 10.1128/mcb.19.3.2322

Barber, K. R., Hruska, M., Bush, K. M., Martinez, J. A., Fei, H., Levitan, I. B., et al. (2018). Levels of Par-1 kinase determine the localization of bruchpilot at the drosophila neuromuscular junction synapses. Sci. Rep. 8:16099. doi: 10.1038/s41598-018-34250-9

Beramendi, A., Peron, S., Casanova, G., Reggiani, C., and Cantera, R. (2007). Neuromuscular junction in abdominal muscles of Drosophila melanogaster during adulthood and aging. J. Comp. Neurol. 501, 498-508. doi: $10.1002 / \mathrm{cne} .21253$

Bergado, J. A., and Almaguer, W. (2002). Aging and synaptic plasticity: a review. Neural Plast. 9, 217-232. doi: 10.1155/NP.2002.217

Bhattacharya, M., Ojha, N., Solanki, S., Mukhopadhyay, C. K., Madan, R., Patel, N., et al. (2006). IL-6 and IL-12 specifically regulate the expression of Rab5 and Rab7 via distinct signaling pathways. EMBO J. 25, 2878-2888. doi: 10.1038/sj.emboj.7601170

Biggs, W. H. III, Zavitz, K. H., Dickson, B., van der Straten, A., Brunner, D., Hafen, E., et al. (1994). The drosophila rolled locus encodes a MAP kinase required in the sevenless signal transduction pathway. EMBO J. 13, 1628-1635.

Birnbaum, A., Wu, X., Tatar, M., Liu, N., and Bai, H. (2019). Age-dependent changes in transcription factor FOXO targeting in female drosophila. Front. Genet. 10:312. doi: 10.3389/fgene.2019.00312

Boaro, S. N., Soares, J. C., and Konig, B. Jr. (1998). Comparative structural analysis of neuromuscular junctions in mice at different ages. Ann. Anat. 180, 173-179. doi: 10.1016/S0940-9602(98)80020-4

Chang, K., Kang, P., Liu, Y., Huang, K., Miao, T., Sagona, A. P., et al. (2019). TGFB-INHB/activin signaling regulates age-dependent autophagy and cardiac health through inhibition of MTORC2. Autograph 16, 1807-1822. doi: 10.1080/15548627.2019.1704117

Colacurcio, D. J., and Nixon, R. A. (2016). Disorders of lysosomal acidification-the emerging role of v-ATPase in aging and neurodegenerative disease. Ageing Res. Rev. 32, 75-88. doi: 10.1016/j.arr.2016.05.004

Correa, S. A., and Eales, K. L. (2012). The role of p38 MAPK and its substrates in neuronal plasticity and neurodegenerative disease. J. Signal. Transduct. 2012:649079. doi: 10.1155/2012/649079

de la Torre-Ubieta, L., Gaudilliere, B., Yang, Y., Ikeuchi, Y., Yamada, T., DiBacco, S., et al. (2010). A FOXO-Pak1 transcriptional pathway controls neuronal polarity. Genes Dev. 24, 799-813. doi: 10.1101/gad.1880510

De Vos, K. J., and Hafezparast, M. (2017). Neurobiology of axonal transport defects in motor neuron diseases: opportunities for translational research? Neurobiol. Dis. 105, 283-299. doi: 10.1016/j.nbd.2017.02.004

Demontis, F., and Perrimon, N. (2010). FOXO/4E-BP signaling in drosophila muscles regulates organism-wide proteostasis during aging. Cell 143, 813-825. doi: 10.1016/j.cell.2010.10.007

Dheen, S. T., Kaur, C., and Ling, E. A. (2007). Microglial activation and its implications in the brain diseases. Curr. Med. Chem. 14, 1189-1197. doi: 10.2174/092986707780597961

Drerup, C. M., and Nechiporuk, A. V. (2013). JNK-interacting protein 3 mediates the retrograde transport of activated c-Jun N-terminal kinase and lysosomes. PLoS Genet. 9:e1003303. doi: 10.1371/journal.pgen.1003303

Ellis, J. E., Parker, L., Cho, J., and Arora, K. (2010). Activin signaling functions upstream of Gbb to regulate synaptic growth at the drosophila neuromuscular junction. Dev. Biol. 342, 121-133. doi: 10.1016/j.ydbio.2010.03.012

Essers, M. A., Weijzen, S., de Vries-Smits, A. M., Saarloos, I., de Ruiter, N. D., Bos, J. L., et al. (2004). FOXO transcription factor activation by oxidative stress mediated by the small GTPase Ral and JNK. EMBO J. 23, 4802-4812. doi: 10.1038/sj.emboj.7600476

Falcicchia, C., Tozzi, F., Arancio, O., Watterson, D. M., and Origlia, N. (2020). Involvement of 38 MAPK in synaptic function and dysfunction. Int. J. Mol. Sci. 21:5624. doi: 10.3390/ijms21165624
Forester, B. P., Berlow, Y. A., Harper, D. G., Jensen, J. E., Lange, N., Froimowitz, M. P., et al. (2010). Age-related changes in brain energetics and phospholipid metabolism. NMR Biomed. 23, 242-250. doi: 10.1002/nbm.1444

Geoffroy, C. G., Meves, J. M., and Zheng, B. (2017). The age factor in axonal repair after spinal cord injury: a focus on neuron-intrinsic mechanisms. Neurosci. Lett. 652, 41-49. doi: 10.1016/j.neulet.2016.11.003

Gibbs, K. L., Kalmar, B., Rhymes, E. R., Fellows, A. D., Ahmed, M., Whiting, P., et al. (2018). Inhibiting p38 MAPK alpha rescues axonal retrograde transport defects in a mouse model of ALS. Cell Death Dis. 9:596. doi: 10.1038/s41419-018-0624-8

Gonzalez-Freire, M., de Cabo, R., Studenski, S. A., and Ferrucci, L. (2014). The neuromuscular junction: aging at the crossroad between nerves and muscle. Front. Aging Neurosci. 6:208. doi: 10.3389/fnagi.2014.00208

Greer, E. L., and Brunet, A. (2005). FOXO transcription factors at the interface between longevity and tumor suppression. Oncogene 24, 7410-7425. doi: 10.1038/sj.onc.1209086

Gwak, Y. S., Hulsebosch, C. E., and Leem, J. W. (2017). Neuronal-glial interactions maintain chronic neuropathic pain after spinal cord injury. Neural Plast. 2017:2480689. doi: 10.1155/2017/2480689

Hall, Z. W., and Sanes, J. R. (1993). Synaptic structure and development: the neuromuscular junction. Cell 72, 99-121. doi: 10.1016/s0092-8674(05)80031-5

Hariharan, N., Maejima, Y., Nakae, J., Paik, J., Depinho, R. A., and Sadoshima, J. (2010). Deacetylation of foxo by sirtl plays an essential role in mediating starvation-induced autophagy in cardiac myocytes. Circ. Res. 107, 1470-1482. doi: 10.1161/CIRCRESAHA.110.227371

He, Y., Yu, W., and Baas, P. W. (2002). Microtubule reconfiguration during axonal retraction induced by nitric oxide. J. Neurosci. 22, 5982-5991. doi: 10.1523/JNEUROSCI.22-14-05982.2002

Hebbar, S., Hall, R. E., Demski, S. A., Subramanian, A., and Fernandes, J. J. (2006). The adult abdominal neuromuscular junction of drosophila: a model for synaptic plasticity. J. Neurobiol. 66, 1140-1155. doi: 10.1002/neu.20279

Hill, C. S., Coleman, M. P., and Menon, D. K. (2016). Traumatic axonal injury: mechanisms and translational opportunities. Trends Neurosci. 39, 311-324. doi: 10.1016/j.tins.2016.03.002

Howlett, E., Lin, C. C., Lavery, W., and Stern, M. (2008). A PI3-kinase-mediated negative feedback regulates neuronal excitability. PLoS Genet. 4:e1000277. doi: 10.1371/journal.pgen.1000277

Hwang, I., Oh, H., Santo, E., Kim, D. Y., Chen, J. W., Bronson, R. T., et al. (2018). FOXO protects against age-progressive axonal degeneration. Aging Cell 17:e12701. doi: 10.1111/acel.12701

Junger, M. A., Rintelen, F., Stocker, H., Wasserman, J. D., Vegh, M., Radimerski, T., et al. (2003). The Drosophila forkhead transcription factor FOXO mediates the reduction in cell number associated with reduced insulin signaling. J. Biol. 2:20. doi: 10.1186/1475-4924-2-20

Kaur, G., and Lakkaraju, A. (2018). Early endosome morphology in health and disease. Adv. Exp. Med. Biol. 1074, 335-343. doi: 10.1007/978-3-319-75402-4_41

Kempsell, A. T., and Fieber, L. A. (2015). Aging in sensory and motor neurons results in learning failure in aplysia californica. PLOS ONE 10:e0127056. doi: 10.1371/journal.pone.0127056

Kent, I. A., Rane, P. S., Dickinson, R. B., Ladd, A. J., and Lele, T. P. (2016). Transient pinning and pulling: a mechanism for bending microtubules. PLoS ONE 11:e0151322. doi: 10.1371/journal.pone.0151322

Kenyon, C., Chang, J., Gensch, E., Rudner, A., and Tabtiang, R. (1993). A C. elegans mutant that lives twice as long as wild type. Nature 366, 461-464. doi: $10.1038 / 366461 \mathrm{a} 0$

Kim, M. J., and O'Connor, M. B. (2014). Anterograde activin signaling regulates postsynaptic membrane potential and GluRIIA/B abundance at the Drosophila neuromuscular junction. PLoS ONE 9:e107443. doi: 10.1371/journal.pone.0107443

Kim, S. Y., and Webb, A. E. (2017). Neuronal functions of FOXO/DAF-16. Nutr. Healthy Aging 4, 113-126. doi: 10.3233/NHA-160009

Kleele, T., Marinkovic, P., Williams, P. R., Stern, S., Weigand, E. E., Engerer, P., et al. (2014). An assay to image neuronal microtubule dynamics in mice. Nat. Commun. 5:4827. doi: 10.1038/ncomms5827

Klinedinst, S., Wang, X., Xiong, X., Haenfler, J. M., and Collins, C. A. (2013). Independent pathways downstream of the Wnd/DLK MAPKKK regulate 
synaptic structure, axonal transport, and injury signaling. J. Neurosci. 33, 12764-12778. doi: 10.1523/JNEUROSCI.5160-12.2013

Koh, Y. H., Ruiz-Canada, C., Gorczyca, M., Budnik, V. (2002). The Ras1mitogen-activated protein kinase signal transduction pathway regulates synaptic plasticity through fasciclin II-mediated cell adhesion. J. Neurosci. 22, 2496-2504. doi: 10.1523/JNEUROSCI.22-07-02496.2002

Li, L., Xiong, W. C., and Mei, L. (2018). Neuromuscular junction formation, aging, and disorders. Annu. Rev. Physiol. 80, 159-188. doi: 10.1146/annurev-physiol-022516-034255

Liu, J., Zhang, B., Lei, H., Feng, Z., Liu, J., Hsu, A. L., et al. (2013). Functional aging in the nervous system contributes to age-dependent motor activity decline in C. elegans. Cell Metab. 18, 392-402. doi: 10.1016/j.cmet.2013.08.007

Lopez-Arias, B., Turiegano, E., Monedero, I., Canal, I., and Torroja, L. (2017). Presynaptic Abeta40 prevents synapse addition in the adult drosophila neuromuscular junction. PLOS ONE 12:e177541. doi: 10.1371/journal.pone.0177541

Lopez-Otin, C., Blasco, M. A., Partridge, L., Serrano, M., and Kroemer, G. (2013). The hallmarks of aging. Cell 153, 1194-1217. doi: 10.1016/j.cell.2013. 05.039

Mahoney, R. E., Azpurua, J., and Eaton, B. A. (2016). Insulin signaling controls neurotransmission via the $4 \mathrm{eBP}$-dependent modification of the exocytotic machinery. Elife 5:16807. doi: 10.7554/eLife.16807

Maiuri, M. C., and Kroemer, G. (2015). Autophagy in stress and disease. Cell Death Differ. 22, 365-366. doi: 10.1038/cdd.2014.236

Manini, T. M., Hong, S. L., and Clark, B. C. (2013). Aging and muscle: a neuron's perspective. Curr. Opin. Clin. Nutr. Metab. Care 16, 21-26. doi: 10.1097/MCO.0b013e32835b5880

Martins, R., Lithgow, G. J., and Link, W. (2016). Long live FOXO: unraveling the role of FOXO proteins in aging and longevity. Aging Cell 15, 196-207. doi: $10.1111 /$ acel.12427

Mattson, M. P., and Magnus, T. (2006). Ageing and neuronal vulnerability. Nat. Rev. Neurosci. 7, 278-294. doi: 10.1038/nrn1886

McLaughlin, C. N., and Broihier, H. T. (2018). Keeping neurons young and foxy: foxos promote neuronal plasticity. Trends Genet. 34, 65-78. doi: 10.1016/j.tig.2017.10.002

McLaughlin, C. N., Nechipurenko, I. V., Liu, N., and Broihier, H. T. (2016). A toll receptor-foxo pathway represses Pavarotti/MKLP1 to promote microtubule dynamics in motoneurons. J. Cell Biol. 214, 459-474. doi: $10.1083 /$ jcb.201601014

McLaughlin, C. N., Perry-Richardson, J. J., Coutinho-Budd, J. C., and Broihier, H. T. (2019). Dying neurons utilize innate immune signaling to prime glia for phagocytosis during development. Dev. Cell. 48, 506-522.e506. doi: 10.1016/j.devcel.2018.12.019

Menon, K. P., Carrillo, R. A., and Zinn, K. (2013). Development and plasticity of the drosophila larval neuromuscular junction. Wiley Interdiscip. Rev. Dev. Biol. 2, 647-670. doi: 10.1002/wdev.108

Milde, S., Adalbert, R., Elaman, M. H., and Coleman, M. P. (2015). Axonal transport declines with age in two distinct phases separated by a period of relative stability. Neurobiol. Aging 36, 971-981. doi: 10.1016/j.neurobiolaging.2014.09.018

Milton, V. J., Jarrett, H. E., Gowers, K., Chalak, S., Briggs, L., Robinson, I. M., et al. (2011). Oxidative stress induces overgrowth of the drosophila neuromuscular junction. Proc. Natl. Acad. Sci. U.S.A. 108, 17521-17526. doi: $10.1073 /$ pnas. 1014511108

Monani, U. R., and De Vivo, D. C. (2014). Neurodegeneration in spinal muscular atrophy: from disease phenotype and animal models to therapeutic strategies and beyond. Future Neurol. 9, 49-65. doi: 10.2217/fnl.13.58

Nechipurenko, I. V., and Broihier, H. T. (2012). Foxo limits microtubule stability and is itself negatively regulated by microtubule disruption. J. Cell Biol. 196, 345-362. doi: $10.1083 /$ jcb. 201105154

Ng, E. L., and Tang, B. L. (2008). Rab GTPases and their roles in brain neurons and glia. Brain Res. Rev. 58, 236-246. doi: 10.1016/j.brainresrev.2008.04.006

$\mathrm{Ng}$, J. (2008). TGF-beta signals regulate axonal development through distinct Smad-independent mechanisms. Development 135, 4025-4035. doi: 10.1242/dev.028209

Nixon, R. A., Yang, D. S., and Lee, J. H. (2008). Neurodegenerative lysosomal disorders: a continuum from development to late age. Autophagy 4, 590-599. doi: $10.4161 /$ auto.6259
Paik, J. H., Ding, Z., Narurkar, R., Ramkissoon, S., Muller, F., Kamoun, W. S., et al. (2009). Foxos cooperatively regulate diverse pathways governing neural stem cell homeostasis. Cell Stem Cell 5, 540-553. doi: 10.1016/j.stem.2009.09.013

Partridge, L., and Barton, N. H. (1996). On measuring the rate of ageing. Proc. R. Soc. Lond. B Biol. Sci. 263, 1365-1371.

Pino, E., Amamoto, R., Zheng, L., Cacquevel, M., Sarria, J.-C., Knott, G. W., et al. (2014). FOXO3 determines the accumulation of alpha-synuclein and controls the fate of dopaminergic neurons in the substantia nigra. Hum. Mol. Genet. 23, 1435-1452. doi: 10.1093/hmg/ddt530

Porras, A., and Mora, F. (1995). Dopamine-glutamate-GABA interactions and ageing: studies in the striatum of the conscious rat. Eur. J. Neurosci. 7, 2183-2188. doi: 10.1111/j.1460-9568.1995.tb00640.x

Punga, A. R., and Ruegg, M. A. (2012). Signaling and aging at the neuromuscular synapse: lessons learnt from neuromuscular diseases. Curr. Opin. Pharmacol. 12, 340-346. doi: 10.1016/j.coph.2012.02.002

Richardson, C. E., Yee, C., and Shen, K. (2019). A hormone receptor pathway cellautonomously delays neuron morphological aging by suppressing endocytosis. PLoS Biol. 17:e3000452. doi: 10.1371/journal.pbio.3000452

Rivlin, P. K., St Clair, R. M., Vilinsky, I., and Deitcher, D. L. (2004). Morphology and molecular organization of the adult neuromuscular junction of drosophila. J. Comp. Neurol. 468, 596-613. doi: 10.1002/cne.10977

Rose, M. R. (1994). Evolutionary Biology of Aging. New York, NY: Oxford University Press on Demand.

Salih, D. A., and Brunet, A. (2008). Foxo transcription factors in the maintenance of cellular homeostasis during aging. Curr. Opin. Cell Biol. 20, 126-136. doi: 10.1016/j.ceb.2008.02.005

Salvadores, N., Sanhueza, M., Manque, P., and Court, F. A. (2017). Axonal degeneration during aging and its functional role in neurodegenerative disorders. Front. Neurosci. 11:451. doi: 10.3389/fnins.2017.00451

Schaffner, I., Minakaki, G., Khan, M. A., Balta, E. A., Schlotzer-Schrehardt, U., Schwarz, T. J., et al. (2018). Foxo function is essential for maintenance of autophagic flux and neuronal morphogenesis in adult neurogenesis. Neuron 99, 1188-1203.e1186. doi: 10.1016/j.neuron.2018.08.017

Schindelin, J., Arganda-Carreras, I., Frise, E., Kaynig, V., Longair, M., Pietzsch, T., et al. (2012). Fiji: an open-source platform for biological-image analysis. Nat. Methods. 9, 676-682. doi: 10.1038/nmeth.2019

Sears, J. C., and Broihier, H. T. (2016). Foxo regulates microtubule dynamics and polarity to promote dendrite branching in drosophila sensory neurons. Dev. Biol. 418, 40-54. doi: 10.1016/j.ydbio.2016.08.018

Segal, M. (1982). Intracellular analysis of a postsynaptic action of adenosine in the rat hippocampus. Eur. J. Pharmacol. 79, 193-199. doi: 10.1016/0014-2999(82)90625-2

Stefanatos, R., and Sanz, A. (2018). The role of mitochondrial ROS in the aging brain. FEBS Lett. 592, 743-758. doi: 10.1002/1873-3468.12902

Takahashi, R. H., Milner, T. A., Li, F., Nam, E. E., Edgar, M. A., Yamaguchi, H., et al. (2002). Intraneuronal alzheimer abeta42 accumulates in multivesicular bodies and is associated with synaptic pathology. Am. J. Pathol. 161, 1869-1879. doi: 10.1016/s0002-9440(10)64463-x

Tang-Schomer, M. D., Patel, A. R., Baas, P. W., and Smith, D. H. (2010). Mechanical breaking of microtubules in axons during dynamic stretch injury underlies delayed elasticity, microtubule disassembly, and axon degeneration. FASEB J. 24, 1401-1410. doi: 10.1096/fj.09-142844

Tank, E. M., Rodgers, K. E., and Kenyon, C. (2011). Spontaneous age-related neurite branching in caenorhabditis elegans. J. Neurosci. 31, 9279-9288. doi: 10.1523/JNEUROSCI.6606-10.2011

Vagnoni, A., and Bullock, S. L. (2018). A cAMP/PKA/Kinesin-1 axis promotes the axonal transport of mitochondria in aging drosophila neurons. Curr. Biol. 28, 1265-1272.e1264. doi: 10.1016/j.cub.2018.02.048

Wagner, N., Laugks, U., Heckmann, M., Asan, E., and Neuser, K. (2015). Aging drosophila melanogaster display altered pre- and postsynaptic ultrastructure at adult neuromuscular junctions. J. Comp. Neurol. 523, 2457-2475. doi: $10.1002 /$ cne. 23798

Wairkar, Y. P., Toda, H., Mochizuki, H., Furukubo-Tokunaga, K., Tomoda, T., and Diantonio, A. (2009). Unc-51 controls active zone density and protein composition by downregulating ERK signaling. J. Neurosci. 29, 517-528. doi: 10.1523/JNEUROSCI.3848-08.2009

Xu, P., Das, M., Reilly, J., and Davis, R. J. (2011). JNK regulates FoxO-dependent autophagy in neurons. Genes Dev. 25, 310-322. doi: 10.1101/gad.1984311 
Yu, L., Hebert, M. C., and Zhang, Y. E. (2002). TGF-beta receptor-activated p38 MAP kinase mediates Smad-independent TGF-beta responses. EMBO J. 21, 3749-3759. doi: 10.1093/emboj/cdf366

Zhan, L., Xie, Q., and Tibbetts, R. S. (2015). Opposing roles of p38 and JNK in a drosophila model of TDP-43 proteinopathy reveal oxidative stress and innate immunity as pathogenic components of neurodegeneration. Hum. Mol. Genet. 24, 757-772. doi: $10.1093 / \mathrm{hmg} / \mathrm{ddu} 493$

Zhao, Y. W., Pan, Y. Q., Tang, M. M., and Lin, W. J. (2018). Blocking p38 signaling reduces the activation of pro-inflammatory cytokines and the phosphorylation of p38 in the habenula and reverses depressive-like behaviors induced by neuroinflammation. Front. Pharmacol. 9:511. doi: 10.3389/fphar.2018.00511
Conflict of Interest: The authors declare that the research was conducted in the absence of any commercial or financial relationships that could be construed as a potential conflict of interest.

Copyright (๑ 2021 Birnbaum, Sodders, Bouska, Chang, Kang, McNeill and Bai. This is an open-access article distributed under the terms of the Creative Commons Attribution License (CC BY). The use, distribution or reproduction in other forums is permitted, provided the original author(s) and the copyright owner(s) are credited and that the original publication in this journal is cited, in accordance with accepted academic practice. No use, distribution or reproduction is permitted which does not comply with these terms. 